Research Article

\title{
Right/Left-Handed Leaky Rectangular Waveguide with Broadside Radiation Property
}

\author{
Xingying $\mathrm{Huo} \mathbb{D}^{1}$ and $\mathrm{Zheng} \mathrm{Li}^{2}$ \\ ${ }^{1}$ Liupanshui Normal University, Liupanshui, China \\ ${ }^{2}$ Beijing Jiaotong University, Beijing, China \\ Correspondence should be addressed to Xingying Huo; hxyandzql@sina.com
}

Received 10 July 2020; Revised 23 December 2020; Accepted 12 January 2021; Published 21 January 2021

Academic Editor: Ping Li

Copyright $\odot 2021$ Xingying Huo and Zheng Li. This is an open access article distributed under the Creative Commons Attribution License, which permits unrestricted use, distribution, and reproduction in any medium, provided the original work is properly cited.

\begin{abstract}
When a leaky rectangular waveguide is used to realize the coverage of radio wave in the small confined spaces, there will be a shadow region, which influences the coverage performance. In this paper, the traditional leaky rectangular waveguide is improved according to the principle of the equivalent circuit, by cutting interdigital slots in the upper wall and adding uniserial metal vias between the upper and lower walls of the rectangular waveguide. Thus, the right/left-handed transmission line property is introduced to the periodic leaky-waveguide (LWG), realizing the broadside radiation with relatively high gain (15.7 dBi), good cross polarization $(-50 \mathrm{~dB})$, and narrow half-power beamwidth $\left(10.9^{\circ}\right)$ at $6.97 \mathrm{GHz}$ and providing a method for a uniform coverage of the radio wave in rooms without a shadow region.
\end{abstract}

\section{Introduction}

With large numbers of mountain railways and railway tunnels built in China, the diversification of the information service is rapidly developed, which requires the real-time and reliable data transmission including pictures, sounds, and environmental parameters. Leaky rectangular waveguide with its simple structure, flexible installation, great capacity of transmission power, low transmission loss at high frequency, and many other advantages has been widely researched and applied in the wireless communication systems [1-10], particularly in some environments where uniform coverage of radio wave is needed, which can replace the traditional distributed antennas [1-6]. In some specific confined spaces, the leaky rectangular waveguide shows much more advantages in realizing uniform and stable radio wave coverage [1-3]. Moreover, in the applications of the $5 \mathrm{G}$ communication systems, the leaky-wave structure even shows tremendous advantages in solving the problems in the aspects of the mutual coupling, isolation degree, and the antenna bandwidth.

However, when the leaky-wave structure is used in the radio wave coverage in the wireless communication systems, the inherent forward-radiation property will produce a shadow region which influences the coverage performance. In order to realize the broadside radiation and the continuous beam-steering, people have been gradually exploring the leaky-wave structures with composite right/left-handed (CRLH) transmission line characteristics in the past years, including designing novel structures and introducing different metamaterial properties, covering the microwave band and even the terahertz band.

In this paper, a periodic leaky-waveguide (LWG) for broadside radiation based on the rectangular waveguide is designed. This proposed periodic LWG can realize the broadside radiation and provide a method to realize the radio wave coverage indoors without a shadow region.

\section{Structure and Mechanism}

The classic hollow metal rectangular waveguide with periodic transverse slots on the upper wall has the forwardradiation property, whose beam direction $\theta$ depends on the ratio between propagation constant $\beta$ and wavenumber in the free space $k_{0}\left(\sin \theta=\beta / k_{0}\right)$, as shown in Figure 1(a). Since 


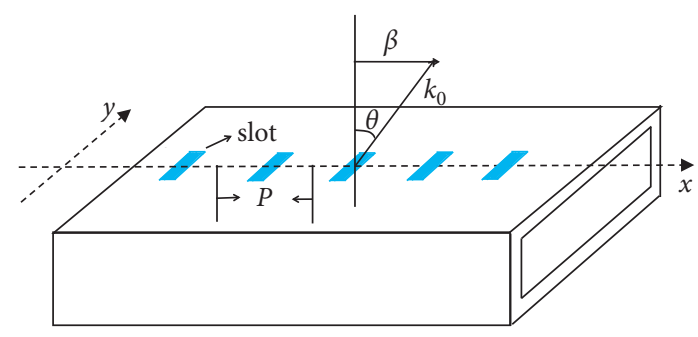

(a)

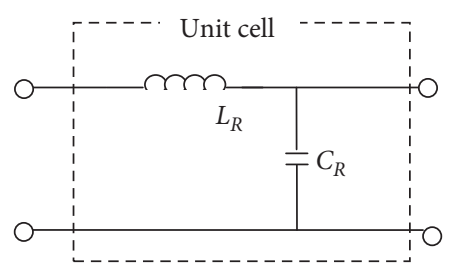

(b)

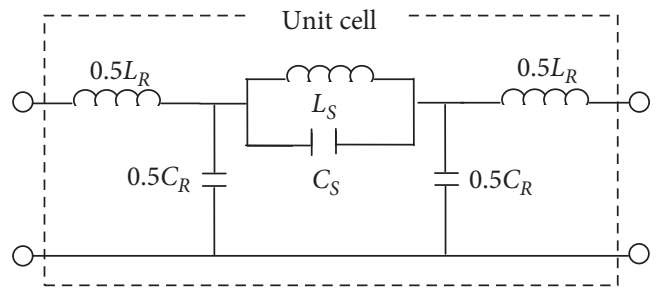

(c)

FIgURE 1: Rectangular waveguide: (a) configuration of whole structure with periodic transverse slots; (b) equivalent circuits for a section of closed rectangular waveguide; (c) equivalent circuits for one period of leaky rectangular waveguide.

this leaky rectangular waveguide is a fast-wave structure and radiates with the fundamental mode, whose propagation constant is smaller than that in free space, its radiation beam always points to the first quadrant. The reason of this inherent right-handed transmission line property could be explained from the circuit parameters. As is known, the equivalent circuit of a section of the closed rectangular waveguide with a length of $P(P$ is smaller than the guide wavelength) consists of a series inductance $L_{R}$ connected with a shunt capacitance $C_{R}$, as shown in Figure 1(b). Generally, the mechanism to realize the right/left-handed transmission line characteristics is to introduce the series capacitance $C_{L}$ and the shunt inductance $L_{L}$ in the rectangular waveguide. As a result, the introduced circuit and the inherent circuit can constitute a new circuit structure, which has the right/left-handed capacitance and inductance at the same time. When the circuit parameters meet the balance conditions, the waveguide can radiate the broadside beam.

In this paper, a novel periodic leaky-wave antenna based on the rectangular waveguide is designed for broadside radiation, as shown in Figure 2(a). It is found that, for periodically slotted rectangular waveguide, the slot itself can be equivalent to the inductance $L_{s}$ and the capacitance $C_{s}[6]$, which are connected in parallel, as shown in Figure 1(c). However, $L_{s}$ is a series inductance and $C_{s}$ is too small to reach balance. In order to introduce the left-handed transmission line property, the interdigital slots are cut on the upper surface to produce enough series capacitance $C_{L}$ [6], and metal vias are set between upper and lower walls to produce enough shunt inductance $L_{L}[10]$.

The transverse interdigital slots and metal vias are periodically set along the waveguide. The structure and parameters of the unit cell are given in Figure 2(b), which is constituted by an interdigital slot and a metal via. This composite interdigital slot consists of a group of transverse and longitudinal rectangular slots, which are intensively arranged to introduce the interdigitated capacitor. The metal vias are set along one side of the waveguide to produce enough shunt inductance. As a result, the equivalent circuit of the unit cell of the proposed structure can be described as that in Figure 2(c).

The dispersion curves of the structure are shown in Figure 3. The dispersion characteristics of the CRLH unit cell have been computed by a full-wave numerical method. A continuous transition at $6.97 \mathrm{GHz}$ is obtained without any gap between the $\mathrm{LH}$ and $\mathrm{RH}$ portions. Thus, the CRLH leaky-wave antenna based on the rectangular waveguide can realize the broadside radiation.

\section{Results}

In this paper, a CRLH LWG based on the rectangular waveguide for broadside radiation is designed based on the standard WR90 rectangular waveguide; the structural parameters are $a=10.15 \mathrm{~mm}, b=22.86 \mathrm{~mm}, A=12.7 \mathrm{~mm}$, and $B=25.4 \mathrm{~mm}$. The details of these parameters of the LWG are shown in Figures 2(a) and 2(b), where $L$ is the whole length of the waveguide, which is set to $120 \mathrm{~mm}$ in this work, and $P$ refers to the period of CRLH unit cell. The performance characteristics of the CRLH LWG were optimized with a series of CST simulations. For broadside radiation, the structural parameters for the unit cell are finally obtained after several trials, as listed in Table 1.

In this work, a CRLH leaky-wave antenna based on the rectangular waveguide with 64 periods is designed for a broadside beam. The simulated radiation patterns in the $H$ plane for the $\mathrm{LH}$ region, broadside, and $\mathrm{RH}$ region are shown in Figures 4(a)-4(c), respectively.

In Figure 4(a), it is found that, by changing the frequency from 6.1 to $6.6 \mathrm{GHz}$, the beam scans from $-20^{\circ}$ to $-5^{\circ}$, with an isotropic gain from $14 \mathrm{dBi}$ to $15.2 \mathrm{dBi}$ and cross polarization below $-30 \mathrm{~dB}$. Similarly, in Figure $4(\mathrm{~b})$, it is found 

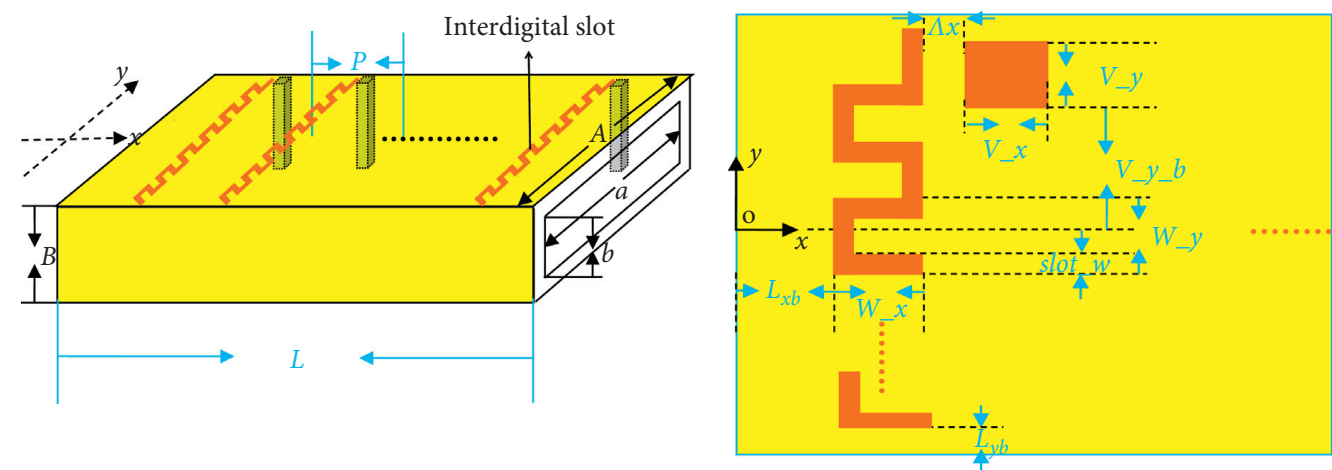

(a)

(b)

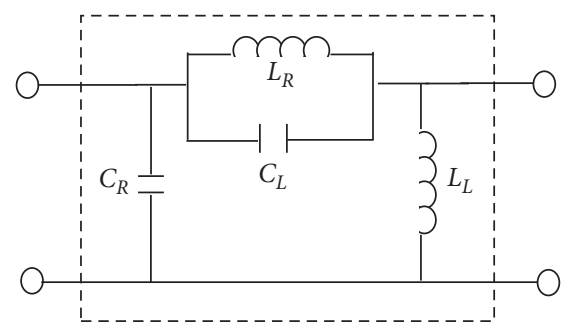

(c)

Figure 2: The proposed periodic leaky rectangular waveguide: (a) perspective view of the whole structure; (b) structure and parameters of the unit cell; (c) equivalent circuit of the unit cell.

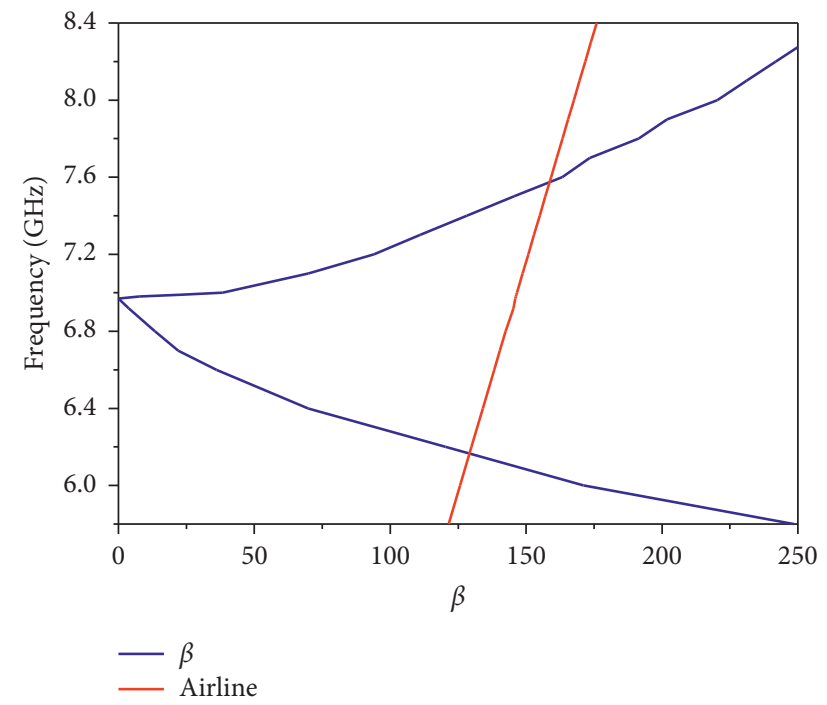

FIGURE 3: Dispersion curves for the proposed CRLH unit cell.

TABLE 1: Design parameter values of the CRLH LWG.

\begin{tabular}{|c|c|}
\hline Parameters & Values $(\mathrm{mm})$ \\
\hline$L_{x b}$ & 1 \\
\hline$L_{y b}$ & 1 \\
\hline$V \_x$ & 2 \\
\hline$V \_y$ & 2 \\
\hline Slot_w & 0.2 \\
\hline$W \_x$ & 0.5 \\
\hline$W_{-} y$ & 0.5 \\
\hline$\Lambda x$ & 1 \\
\hline$V_{-} y_{-} b$ & 10 \\
\hline$P$ & 3 \\
\hline
\end{tabular}




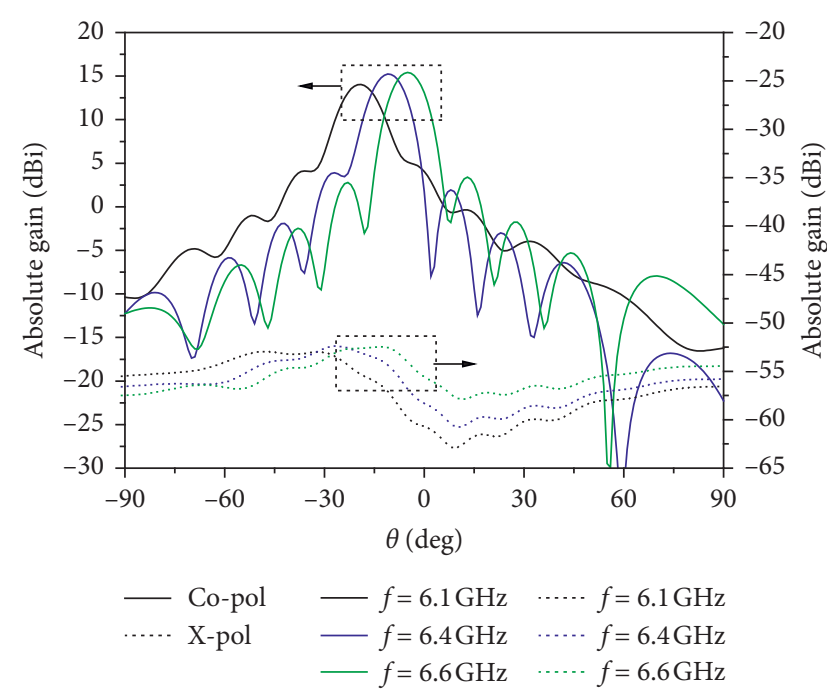

(a)

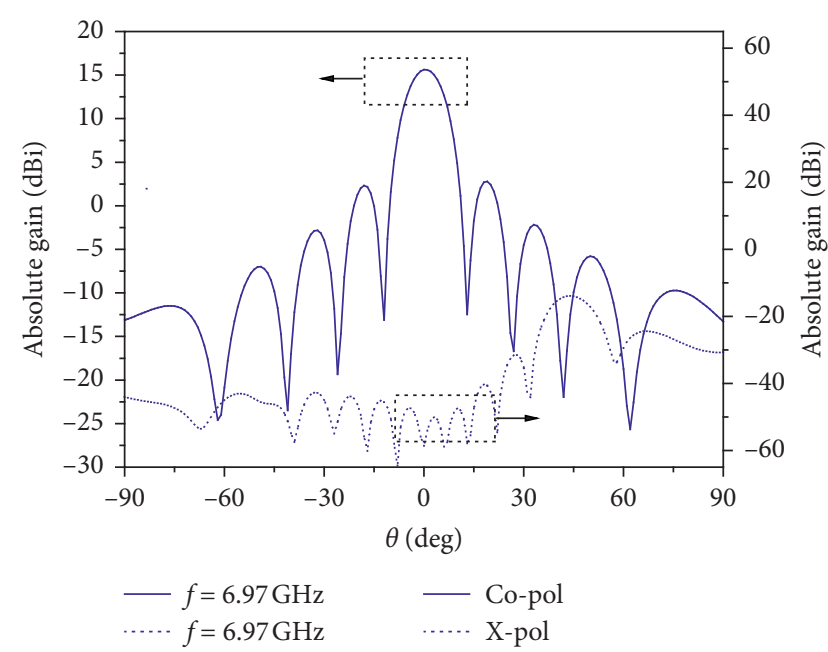

(b)



(c)

FIgURE 4: Simulated radiation patterns in the $H$-plane: (a) LH region; (b) broadside; (c) RH region.

that, by changing the frequency from 7.0 to $7.6 \mathrm{GHz}$, the beam scans from $5^{\circ}$ to $18^{\circ}$, with an isotropic gain from $15.2 \mathrm{dBi}$ to $14.4 \mathrm{dBi}$ and cross polarization below $-35 \mathrm{~dB}$. In Figure 4(c), it shows that when the LWG works at $6.97 \mathrm{GHz}$, the beam points to the broadside and the realized gain can reach to $15.7 \mathrm{dBi}$ with the cross polarization below $-50 \mathrm{dBi}$.

At the same time, the simulated radiation patterns for seven operating frequencies of the LWG show that the halfpower beamwidths (HPBWs) of the backward and forward beams are wider than this of the broadside beam. For example, the HPBW is $14^{\circ}$ for $6.1 \mathrm{GHz}$ and is $10.9^{\circ}$ for $6.97 \mathrm{GHz}$.

Figure 5 shows the simulated $S$-parameters as functions of the frequency. It is seen that the $S_{11}$ fluctuates around $-10 \mathrm{~dB}$ in the $\mathrm{LH}$ region except for the transition frequency $(6.97 \mathrm{GHz})$ and keeps below $-10 \mathrm{~dB}$ in the $\mathrm{RH}$ region. At the same time, the values of $S_{21}$ increase in the $\mathrm{LH}$ region and become almost stable in the $\mathrm{RH}$ region. As a result, the radiation efficiency decreases in the LH region and increases

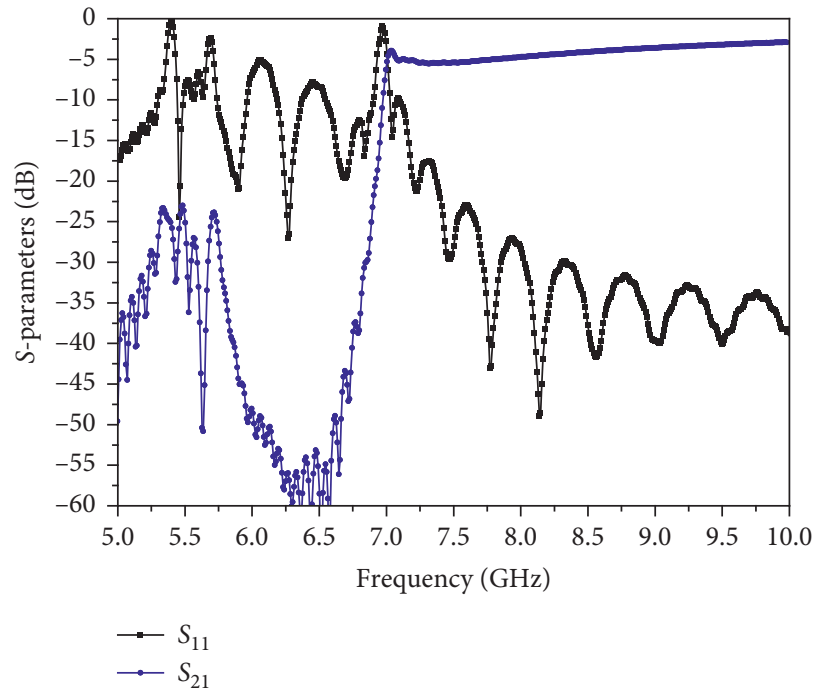

FIGURE 5: Scattering parameters for the proposed CRLH LWG. 
in the $\mathrm{RH}$ region. Also, it could be figured out that the radiation efficiency is around $70 \%$ within the whole scanning range.

\section{Conclusion}

In this paper, a periodic leaky-wave antenna based on the rectangular waveguide is proposed to radiate a broadside beam for radio wave coverage in the confined space. The interdigital slots and metal vias are designed to introduce the left-handed transmission line properties, and the radiating mechanism is investigated as well. The LWG can radiate a broadside beam at $6.97 \mathrm{GHz}$, with relatively high gain $(15.7 \mathrm{dBi})$ and narrow half-power beamwidth $\left(10.9^{\circ}\right)$. Performance degradation in the scattering parameters is observed around the transition frequency. Further improvement may be required to introduce the transition feeding structure. Besides, it is not easy to fabricate the composite slots accurately on the metal surface. Thus, a similar CRLH LWG based on the dielectric waveguide will be further studied to replace the metal structure. However, the periodic CRLH LWG based on the rectangular waveguide in this article shows a low cross polarization $(-50 \mathrm{dBi})$ and relatively high gain $(15.7 \mathrm{dBi})$ compared to those in the previous work $(-10 \mathrm{dBi}$ and $12.5 \mathrm{dBi}$, respectively) [11], where the LWG is $20 \mathrm{~mm}$ longer than that in this paper.

\section{Data Availability}

The data used to support the findings of this study are included within the article.

\section{Conflicts of Interest}

The authors declare that there are no conflicts of interest regarding the publication of this paper.

\section{Acknowledgments}

This work was supported in part by the National Natural Science Foundation of China (Grant no. 61771038), in part by the National Science Research Project of Department of Education in Guizhou Province (Grant nos. KY[2019]125, $\mathrm{KY}[2019] 144, \mathrm{KY}[2020] 112$, and $\mathrm{KY}[2020] 062)$, in part by the High-level Talent Starting Foundation of Liupanshui Normal University (Grant no. LPSSYKYJJ201802), and in part by the Foundation of LiuPanShui Technology Council (Grant no. 52020-2018-04-04).

\section{References}

[1] X. Huo, J. Wang, D. Li, Z. Zhang, M. Chen, and Z. Li, "Leaky rectangular waveguide with circular polarization property," IEEE Transactions on Antennas and Propagation, vol. 63, no. 11, pp. 5098-5101, 2015.

[2] L. Li-Si, J.-H. Wang, Z. Zhang et al., "Radiation characteristics of rectangular leaky waveguide under high speed railway environments," in Proceedings of the 4th IEEE International Symposium on Microwave, Antenna, Propagation, and EMC Technologies for Wireless Communications, Beijing, China, November 2011.
[3] X.-Y. Huo, J.-H. Wang, and L. Da-Wei, "Effects of structural and environmental parameters on the coupling loss of leaky rectangular waveguide in tunnel," IEEE Loughborough Antennas and Propagation Conference, Loughborough, Britain, vol. 34, 2015.

[4] C. Alcala, S.-Y. Lin, R.-S. He et al., "Design and test of a high QoS radio network for CBTC systems in subway tunnels," 2011.

[5] M. Garcia-Vigueras, J. L. Gomez-Tornero, G. Goussetis, A. R. Weily, and Y. J. Guo, "Enhancing frequency-scanning response of leaky-wave antennas using high-impedance surfaces," IEEE Antennas and Wireless Propagation Letters, vol. 10, pp. 7-10, 2011.

[6] X. Huo, J. Wang, D. Li, Z. Zhang, M. Chen, and Z. Li, "Research on the propagation constant of the periodically slotted rectangular waveguide by equivalent circuit method," Microwave and Optical Technology Letters, vol. 58, no. 2, pp. 426-429, 2016.

[7] T. Kodera and C. Caloz, "Uniform ferrite-loaded open waveguide structure with CRLH response and its application to a novel backfire-to-endfire leaky-wave antenna," IEEE Transactions on Microwave Theory and Techniques, vol. 57, no. 4, pp. 784-795, 2009.

[8] T. Ikeda, K. Sakakibara, T. Matsui, N. Kikuma, and H. Hirayama, "Beam-scanning performance of leaky-wave slot-array antenna on variable stub-loaded left-handed waveguide," IEEE Transactions on Antennas and Propagation, vol. 56, no. 12, pp. 3611-3618, 2008.

[9] Y. Chen, S.-W. Liao, J. Wei, and J.-H. Xu, "Unequally spaced and excited resonant slotted-waveguide antenna array based on an improved resonant-slot coupled cavity chain composite right/left-handed waveguide," Progress in Electromagnetics Research, vol. 110, pp. 421-435, 2010.

[10] C. Jin, A. Alphones, and M. Tsutsumi, "Double periodic composite right/left handed transmission line and its applications to compact leaky-wave antennas," IEEE Transactions on Antennas and Propagation, vol. 59, no. 10, pp. 3679-3686, 2011.

[11] Y. kushiyama, T. Arima, and T. Uno, "Differential-type CRLH leaky-wave antenna using stepped impedance resonators," IEEE Antennas and Wireless Propagation Letters, vol. 15, pp. 321-324, 2016. 